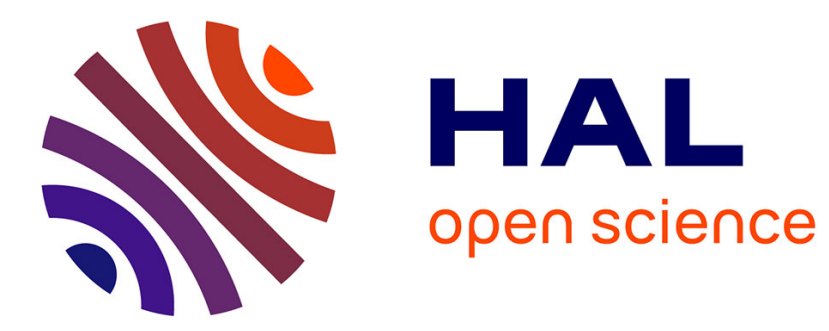

\title{
Exemplar-based colorization in RGB color space
}

Fabien Pierre, Jean-François Aujol, Aurélie Bugeau, Nicolas Papadakis, Vinh-Thong Ta

\section{To cite this version:}

Fabien Pierre, Jean-François Aujol, Aurélie Bugeau, Nicolas Papadakis, Vinh-Thong Ta. Exemplarbased colorization in RGB color space. IEEE Internationnal Conference on Image Processing (ICIP'14), Oct 2014, Paris, France. pp.1-5. hal-01019627

\section{HAL Id: hal-01019627 https://hal.science/hal-01019627}

Submitted on 7 Jul 2014

HAL is a multi-disciplinary open access archive for the deposit and dissemination of scientific research documents, whether they are published or not. The documents may come from teaching and research institutions in France or abroad, or from public or private research centers.
L'archive ouverte pluridisciplinaire HAL, est destinée au dépôt et à la diffusion de documents scientifiques de niveau recherche, publiés ou non, émanant des établissements d'enseignement et de recherche français ou étrangers, des laboratoires publics ou privés. 


\title{
EXEMPLAR-BASED COLORIZATION IN RGB COLOR SPACE.
}

\author{
Fabien Pierre ${ }^{1,2,3,4}$, Jean-François Aujol ${ }^{1,2}$, Aurélie Bugeau ${ }^{3,4}$, Nicolas Papadakis ${ }^{1,2}$, Vinh-Thong Ta ${ }^{4,5}$ \\ ${ }^{1}$ Univ. Bordeaux, IMB, UMR 5251, F-33400 Talence, France. \\ ${ }^{2}$ CNRS, IMB, UMR 5251, F-33400 Talence, France. \\ ${ }^{3}$ Univ. Bordeaux, LaBRI, UMR 5800, F-33400 Talence, France. \\ ${ }^{4}$ CNRS, LaBRI, UMR 5800, F-33400 Talence, France. \\ ${ }^{5}$ IPB, LaBRI, UMR 5800, F-33600 Pessac, France.
}

\begin{abstract}
This paper deals with the problem of image colorization. A model including total variation regularization is proposed. Our approach colorizes directly the three RGB channels, while most existing methods were only focusing on the two chrominance channels. By using the three channels, our approach is able to better preserve color consistency. Our model is non convex, but we propose an efficient primal-dual like algorithm to compute a local minimizer. Numerical examples illustrate the good behavior of our algorithm with respect to state-of-the-art methods.
\end{abstract}

Index Terms - Colorization, total variation, patches, optimization, variational methods.

\section{INTRODUCTION}

Image colorization is an old research field that started in 1970 with Wilson Markle (see details in Levin et al. [2]). It appeared naturally for restoration of old documents and movies. The objective is to transform a given gray-scale image (called target) into a colored one. As no color information is present in a gray-scale image, additional prior is needed. It can be done in two ways: with manual interactions or by giving a color image as an example.

In manual methods (following the work of [2]), the user defines colors on some points of the image and an algorithm is used to spatially diffuse the color. These methods have a main drawback: if the image represents a complex scene, the user has to draw a lot of seeds. In exemplar-based colorization methods, the color information is extracted from a color image, called source, selected by the user. Figure 1 shows an example of such a method where the final result is obtained with the approach introduced in this paper.

This study has been carried out with financial support from the French State, managed by the French National Research Agency (ANR) in the frame of the Investments for the future Programme IdEx Bordeaux (ANR10-IDEX-03-02). J-F. Aujol is a member of Institut Universitaire de France. The authors would like to thank Raj Kumar Gupta for providing the images presented in [1].

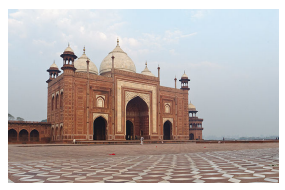

(a) Source.

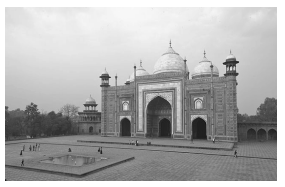

(b) Target.

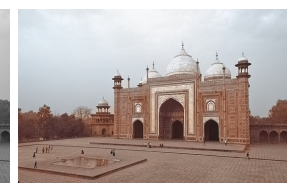

(c) Our result.
Fig. 1. Exemplar-based image colorization.

The first exemplar-based method is the one proposed by Welsh et al. [3]. It is derived from the texture synthesis algorithm of Wey et al. [4] which uses image patch similarities on the intensity channel to provide colors. Such methods suffer from spatial consistency since each pixel is processed independently. Hence, Irony et al. [5] considered the diffusion step of [2] as a post-processing, in order to regularize the colors given by the exemplar-based approach. Recently, Gupta et al. [1] segments the image in order to guide the selection of examples and regularize the colors with [2]. The approach of Charpiat et al. [6] ensures a spatial coherency without a segmentation but involves many complex steps while Chen et al. [7] uses image matting. Finally, Bugeau et al. [8] presents a framework for exemplar-based colorization based on the minimization of a functional including a total variation (TV) regularization on the chrominance channels. Nevertheless theirs results are too drab, in particular near the contours, and a transformation in the $Y U V$ space is needed which can create colors that do not exist in the example images.

In this paper, we propose an extension of [8] that overcomes these issues. Our new model differs from the chrominance model [8] which suffers from a lack of coupling between the luminance $Y$ and the chrominance channels ( $U$ and $V$ ) during the regularization. In our model the colorization is done directly in the $R G B$ space with coupled channels. This permits to preserve colors, in particular near the contours. Up to our knowledge, our model is the first exemplar-based colorization algorithm using the $R G B$ color space. Notice that the $R G B$ space is only used in [9], [10], which are manual methods.

The paper is organized as follows: we first review the 
chrominance model [8], then we present our new model with an efficient primal-dual like algorithm [11] minimizing the functional. Finally, the behavior of the method is presented with comparisons with the state-of-the-art methods.

\section{REVIEW OF THE CHROMINANCE MODEL [2]}

The authors of [8] chose to work in the $Y U V$ space in order to easily constrain the $Y$ channel to be equal to the original target image. Hence, all the information present in the gray-scale image are preserved, i.e., contours or textures. The method consists in only computing the two chrominance channels ( $U$ and $V$ ) of the colorized image. Together with the $Y$ channel, it permits to recover a $R G B$ image.

The method starts by extracting, for each pixel of the target image, 8 chrominance candidates $c_{i}$ with $i=1, \cdots, 8$ by comparing patches around pixel and using different features (variance, cumulative histogram and magnitude of the DFT for different sizes of patches). To select one color between these 8 candidates, an energy-based method is proposed. To ensure the regularity of the resulting image $u$ defined on the domain $\Omega$, the model includes a TV regularization of the $U$ and $V$ channels. Let $u=(U, V)$ be the chrominance to compute and $W=\left\{w_{i}\right\}$ be the candidate weights, the model of [8] reads:

$$
\begin{aligned}
& \begin{aligned}
F_{1}(u, W) & :=T V(u)+\lambda / 2 \int_{\Omega} \sum_{i=1, \cdots, 8} w_{i}\left\|u-c_{i}\right\|^{2} \\
& +\alpha \int_{\Omega} \sum_{i=1, \cdots, 8} w_{i}\left(1-w_{i}\right)+\chi_{u \in \mathcal{R}}+\chi_{W \in \Delta}
\end{aligned} \\
& \text { where } T V(u)=\int_{\Omega} \sqrt{\sum_{C=U, V} \partial_{x} C^{2}+\partial_{y} C^{2}} \\
& \text { and } \Delta:=\left\{\left(w_{1}, \cdots, w_{8}\right) \text { st } 0 \leq w_{i} \leq 1 \text { and } \sum_{i} w_{i}=1\right\} .
\end{aligned}
$$

The set $\mathcal{R}$ is the standard range for the chrominance and the characteristic function $\chi_{u \in X}$ is 0 if $u \in X$ and $\infty$ otherwise. The model is continuous, and the fidelity-data term is the transformation of a labeling problem into a continuous term by introducing a weight $w_{i}$ corresponding to the probability of considering the color candidate $c_{i}$. The term $\int_{\Omega} \sum_{i=1}^{8} w_{i}\left\|u-c_{i}\right\|^{2}$ makes the link between the candidate color $c_{i}$ and the color $u$ which will be retained. Each candidate is weighted, and the fidelity-data term can be close to a melting of two colors, which does not correspond to any color of the source image. To tackle this issue the weights $w_{i}$ are forced to be close to 0 or 1 with the sum equal to 1 . To this end, the term $\int_{\Omega} \sum_{i=1}^{8} w_{i}\left(1-w_{i}\right)$ is introduced. $\lambda$ and $\alpha$ are parameters that weight the influence of the different terms of the model. The functional is not convex and may admit local minima.

\section{RGB MODEL FOR COLORIZATION}

The results presented in [8] are visually good but the methods still presents some drawbacks. The resulting images are drab since if a strong regularization is used, the $U$ and $V$ channels become constant, and the image is close to a gray-scale one. Another problem which naturally arises is the lack of coupling in direction between color channels creating halos near strong contours. To couple the three color channels, we propose to work directly with the $R G B$ space.

The chrominance model [8] is invariant with respect to the scene illumination but the method only retains the $U$ and $V$ values. The consequence is: with a given $U$ and $V$, different colors can be obtained when varying $Y$ (see Figure 2) and can produce colors with hues that are not present in the candidates data. It is therefore preferable to directly work with the three

Fig. 2. If $U$ and $V$ are constant, and $Y$ variates, different colors are obtained.

channels. In this paper, we propose a $R G B$-model that is invariant with respect to the illumination. A main advantage of our approach is that a color space transformation at the beginning and the end of the algorithm is not necessary. In order to take into account all these elements, we introduce the following functional, where $u$ is now a $R G B$ image:

$$
\begin{aligned}
& F_{2}(u, W):=T V_{R G B}(u)+\lambda / 2 \int_{\Omega} \sum_{i=1, \cdots, 8} w_{i}\left\|u-c_{i}\right\|^{2} \\
& \quad+\alpha \int_{\Omega} \sum_{i=1, \cdots, 8} w_{i}\left(1-w_{i}\right) \\
& \quad+\chi_{u \in[0,255]^{3}}+\chi_{Y(u)=I_{g}}+\chi_{W \in \Delta} \\
& \text { where } T V_{R G B}(u)=\int_{\Omega} \sqrt{\sum_{C=R, G, B} \partial_{x} C^{2}+\partial_{y} C^{2}} .
\end{aligned}
$$

Two constraints are added to the original model: the colorized image should be between 0 and 255 and the second constraint is that the luminance of the colorized image should be the same as the target gray-scale image in order to preserve image textures. The luminance constraint is given by $Y(u)=$ $A$. $u=I_{g}$ where $u=(R, G, B), I_{g}$ is the original luminance of the target image, and $A=(0.2990,0.5870,0.1140)$ allows recovering the luminance of a $R G B$ color.

Minimization of the functional. In order to estimate a local minimum of (4), we consider a primal-dual algorithm [11] with respect to the variable $u$ and a projected gradient update for the variable $W=\left\{w_{i}\right\}$ with time step $\tau_{w}>0$. The process is summarized in Algorithm 1. The dual variable $Z \in \mathbb{R}^{6}$ is related to the TV regularization in the $R G B$ space and $P_{B}$ is the projection onto the unit ball of $\mathbb{R}^{6} . P_{\Delta}$ is the projection onto the simplex $\Delta$ defined by (3) that can be computed using [12]. The term $\left(\left\|u-c_{i}\right\|^{2}\right)_{i}$ represents the array of the same size of $W$ such that each weight is equal to $\left\|u(x)-c_{i}(x)\right\|^{2}$ for $i \in 1, \cdots, 8$ and position $x \in \Omega$. Notice that if $W$ is fixed, then the model is convex in $u$ and the algorithm converges [13] if $24 \tau \sigma<1$.

The problem of luminance and range. The natural problem that arises when we want to implement the primal-dual algo- 

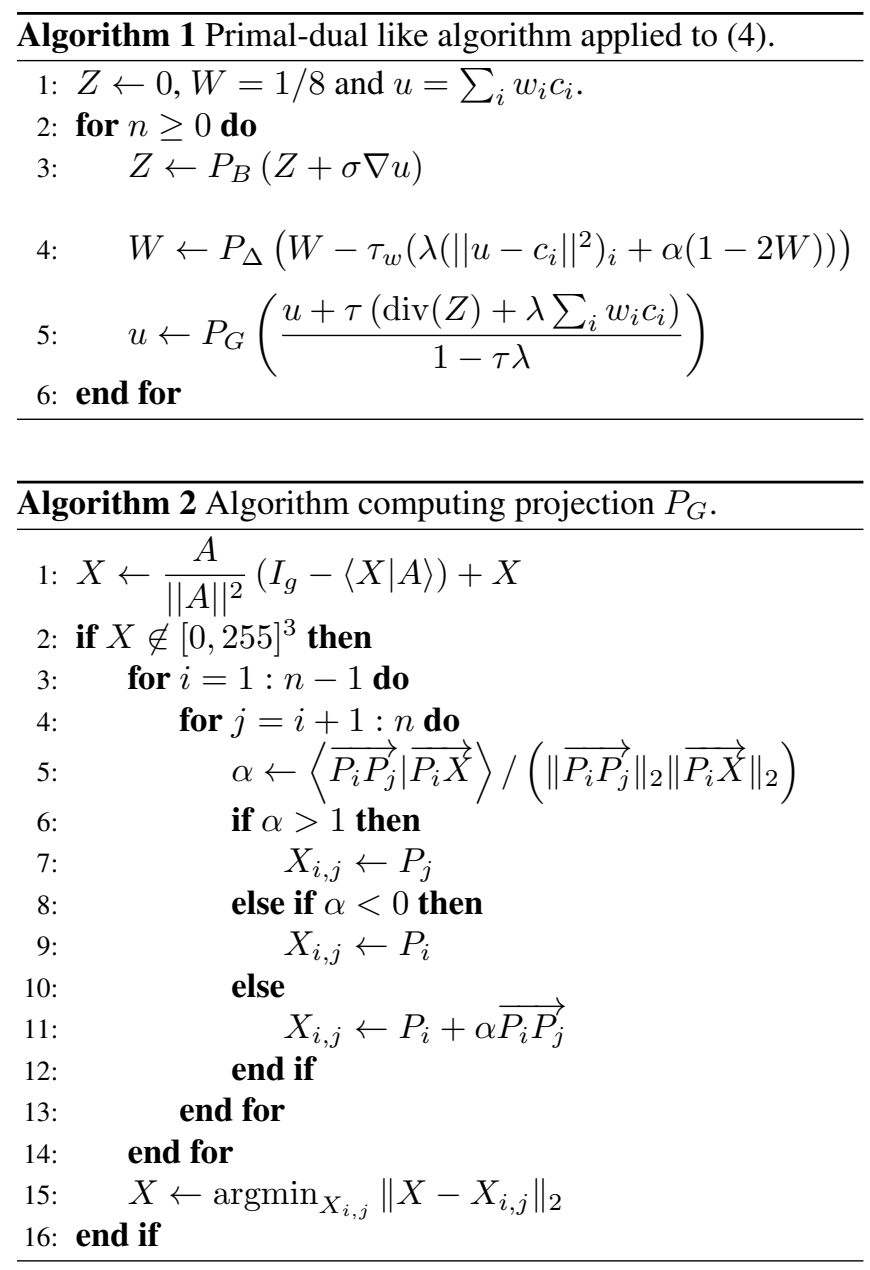

rithm is the projection of $u$ onto its constraints through the operator $P_{G}$, i.e., the computation of the proximal operator of $\chi_{u \in[0,255]^{3}}+\chi_{Y(u)=I_{g}}$. This is equivalent to computing the projection onto the intersection of the cube $[0,255]^{3}$ and the affine plane defined by $A . u=I_{g}$. There are four cases for this set. It can be a singleton, a triangle, a quadrilateral or a pentagon. To compute the projection we proceed in two steps. First, we compute the projection onto the plane, then we project onto the intersection of the cube and the plane. The projection of a point onto the intersection of the cube $[0,255]^{3}$ and the affine plane defined by $A . u=I_{g}$ is given in Algorithm 2, assuming this intersection is a polygon defined by points $P_{1}, \cdots, P_{n}$.

The main drawback of this projection is the change of hue of the color (the $H$ channel of the $H S I$ color space [14]) during the computation, because the plane $\{Y=$ constant $\}$ is not orthogonal to the axis $I$ oriented from the white to the black. In practice, the $H$ channel is maintained constant during the projection by slightly modifying Algorithm 2 in order to consider an oblique projection that preserve the hue. For the sake of simplicity, this projection is not detailed here (see [15] or [16] for more details).

\section{NUMERICAL RESULTS}

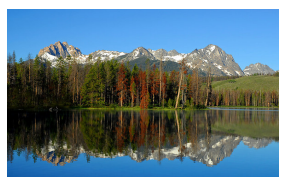

(a) Source image.

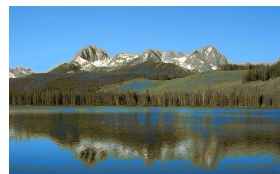

(c) Initialization

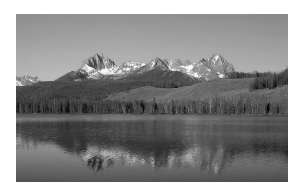

(b) Target image.

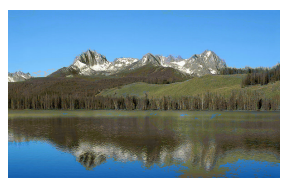

(d) Final labeling

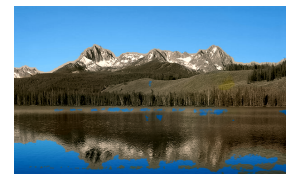

(e) Result with [8].

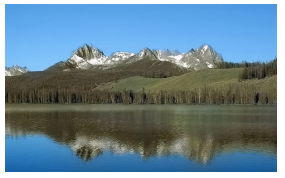

(f) Our result.

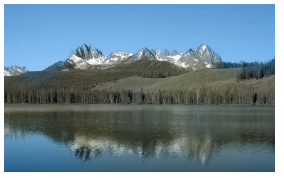

(g) Our result with strong regularization.
Fig. 3. Results with different regularization, with initialization and with data term at convergence. See text for details.

Figure 3 shows the details of a first colorization. Figure 3(c) is the initialization of the algorithm: $u=\sum_{i} c_{i} / 8$. The result obtained by our method is presented in Figure 3(f). Resulting colors are visually close to the ones of the source image (Figure 3(a)). Our approach improves visually the result obtained with the chrominance model [8] (Figure 3(e)). Indeed, the contours with our method are better preserved and we are able to recover some green on the hills. This confirms that the regularization term must couple all the three channels of color to have a good preservation of shapes. It is worth mentioning that our algorithm also provides a regularized labeling of the discrete original problem which is the choice of a candidate for each pixel. This is illustrated in Figure 3(d) that presents the labeling image $u=\sum_{i} w_{i} c_{i}$ after convergence of the algorithm. Finally notice that the images are drab when a strong regularization is used $\left(\lambda=10^{-7}\right)$, see Figure $3(\mathrm{~g})$. If the regularization is weaker, the images are shinier and visually close to the obtained labeling (Figure 3(d)). In natural images a good parameter is about $7 \cdot 10^{-3}$.

The minimization of the functional with respect to $u$ improves the quality of contours and prevents stains in constant regions of the image. Figure 4 shows experimental results compared to the state-of-the-art methods. On the left, the source and target images are shown. Our results are in the third column and other columns are results from [1], [5], [3] and [6] (these results have been directly taken in the article [1]). The results of [5] and [6] are not good although their algorithms need many steps. Due to the lack of regularization, images of [3] present artefacts where areas that were originally homogeneous now present irregularities. Our algorithm better preserves the homogeneous parts as the sky. 

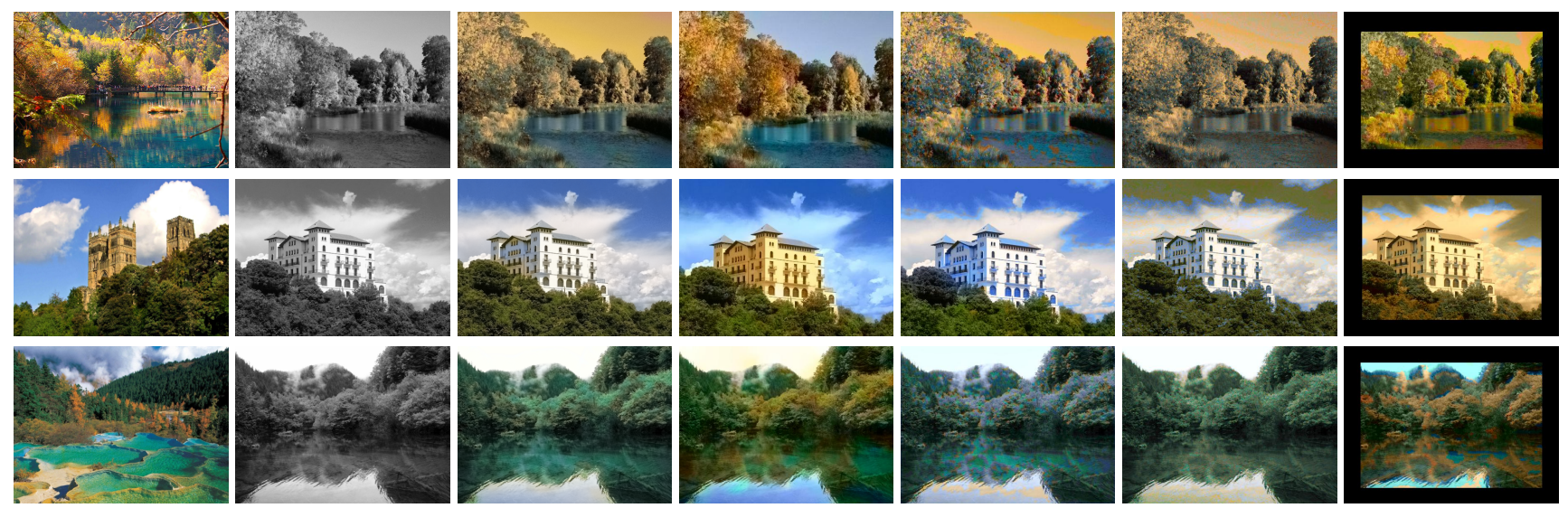

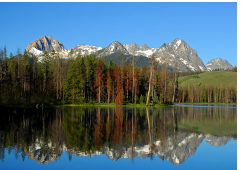

Source.

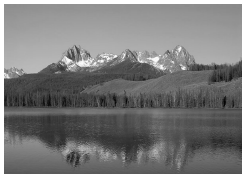

Target.

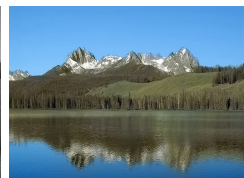

Our result.

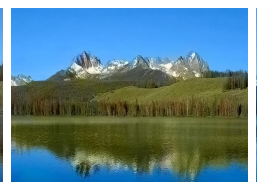

[1].

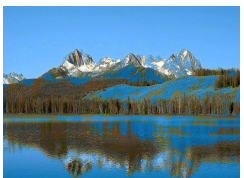

[5].

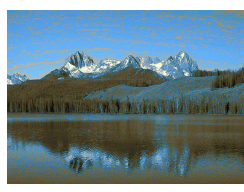

[3].

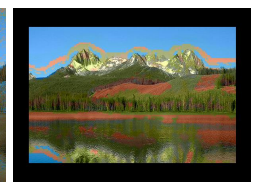

[6].

Fig. 4. Comparison with state-of-the-art methods. On the left, the source and the target images. Results of our method on four images, compared with the results of the state-of-the-art methods [1], [5], [3] and [6] (images taken from [1]).

The quality of our results are comparable to [1] whereas our algorithm is much simpler since local segmentation like superpixels [17] is not needed. Currently, our is faster, but we expect to speed-up it more with discrete optimization methods.
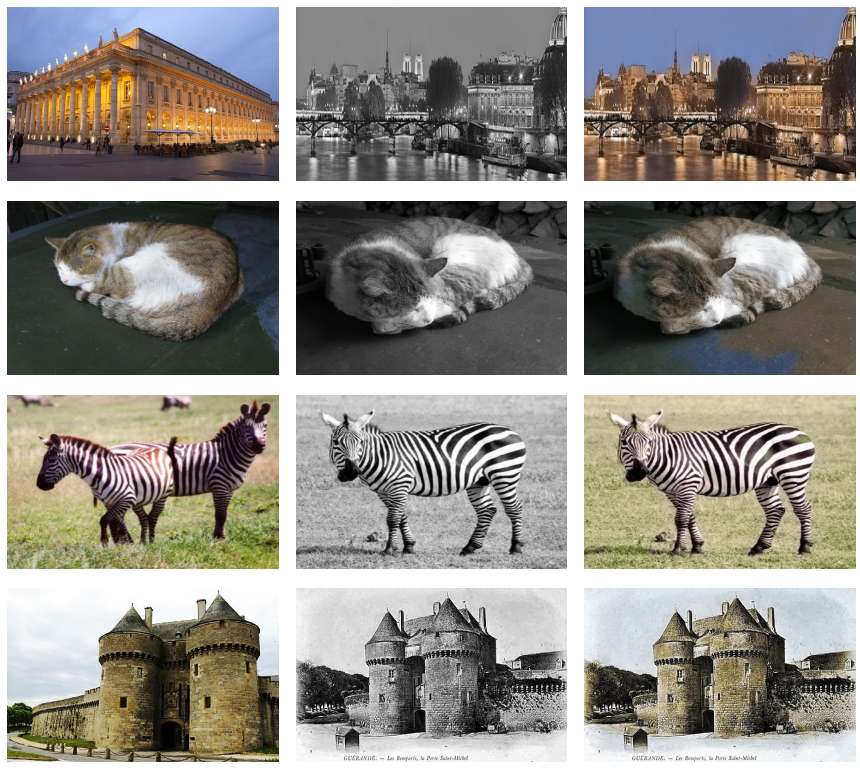

Fig. 5. Additional results.

Figure 5 present additional results. They show that our method is efficient and competitive in order to colorize grayscale images with examples. Finally, Figure 6 highlight the influence of the source image on the colorization result. Us-
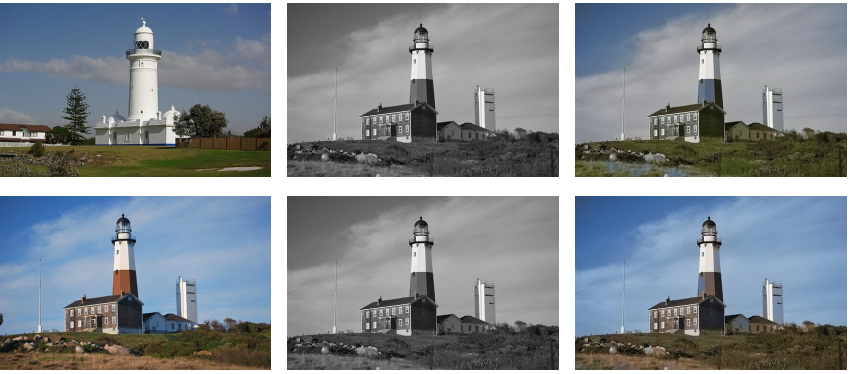

Fig. 6. Influence of the source image.

ing several source images could improve the quality of all the results and is directly feasible with the described method.

\section{CONCLUSION AND FUTURE WORKS}

In this paper a new model for exemplar-based colorization in the $R G B$ color space has been described. The regularization is provided by the minimization of the coupled total variation of color channels. An efficient algorithm solving the proposed model has been given. This new approach extends and improves the chrominance model [8]. The resulting images are less drab, the color are better preserved and the contours are well colorized. In futur works, we plan to extend the method to integrate manual colorization, and to investigate the choice of good metrics for searching color candidates. 


\section{REFERENCES}

[1] Raj Kumar Gupta, Alex Yong-Sang Chia, Deepu Rajan, Ee Sin Ng, and Huang Zhiyong, "Image colorization using similar images," in ACM International Conference on Multimedia, 2012, pp. 369-378.

[2] Anat Levin, Dani Lischinski, and Yair Weiss, "Colorization using optimization," in ACM Transactions on Graphics, 2004, vol. 23, pp. 689-694.

[3] Tomihisa Welsh, Michael Ashikhmin, and Klaus Mueller, "Transferring color to greyscale images," ACM Transactions on Graphics, vol. 21, no. 3, pp. 277-280, 2002.

[4] Li-Yi Wei and Marc Levoy, "Fast texture synthesis using tree-structured vector quantization," in ACM Computer graphics and interactive techniques. Press/AddisonWesley Publishing Co., 2000, pp. 479-488.

[5] Revital Irony, Daniel Cohen-Or, and Dani Lischinski, "Colorization by example," in Eurographics conference on Rendering Techniques. Eurographics Association, 2005, pp. 201-210.

[6] G. Charpiat, M. Hofmann, and B. Schölkopf, "Automatic image colorization via multimodal predictions," in European Conference on Computer Vision, D. Forsyth, P. Torr, and A. Zisserman, Eds., Marseille, France, 10 2008, pp. 126-139, Springer.

[7] Tongbo Chen, Yan Wang, Volker Schillings, and Christoph Meinel, "Grayscale image matting and colorization," in Asian Conference on Computer Vision, 2004, pp. 1164-1169.

[8] Aurélie Bugeau, Vinh-Thong Ta, and Nicolas Papadakis, "Variational exemplar-based image colorization," IEEE Transactions on Image Processing, vol. 23, no. 1, pp. 298-307, 2014.

[9] Takahiko Horiuchi, "Colorization algorithm using probabilistic relaxation," Image and Vision Computing, vol. 22, no. 3, pp. 197-202, 2004.

[10] Tomohisa Takahama, Takahiko Horiuchi, and Hiroaki Kotera, "Improvement on colorization accuracy by partitioning algorithm in cielab color space," in Advances in Multimedia Information Processing-PCM 2004, pp. 794-801. Springer, 2005.

[11] Antonin Chambolle and Thomas Pock, "A first-order primal-dual algorithm for convex problems with applications to imaging," Journal of Mathematical Imaging and Vision, vol. 40, no. 1, pp. 120-145, 2011.

[12] Yunmei Chen and Xiaojing Ye, "Projection onto a simplex," arXiv preprint arXiv:1101.6081, 2011.
[13] Antonin Chambolle, "An algorithm for total variation minimization and applications," Journal of Mathematical Imaging and Vision, vol. 20, no. 1-2, pp. 89-97, 2004.

[14] Rafael C. Gonzales and Richard E. Woods, Digital image processing, 1993, Addison-Wesley Publishing Company.

[15] Fabien Pierre, Jean-François Aujol, Aurélie Bugeau, Vinh-Thong Ta, and Nicolas Papadakis, "Hue constrained image colorization in the RGB space," In preparation., 2014.

[16] Mila Nikolova and Gabriele Steidl, "Fast hue and range preserving histogram specification: Theory and new algorithms for color image enhancement," Preprint, 2013.

[17] Alex Levinshtein, Adrian Stere, Kiriakos N. Kutulakos, David J. Fleet, Sven J Dickinson, and Kaleem Siddiqi, "Turbopixels: Fast superpixels using geometric flows," IEEE Transactions on Pattern Analysis and Machine Intelligence, vol. 31, no. 12, pp. 2290-2297, 2009. 